\title{
Abkommenswidrige Besteuerung deutscher Ärzte in der Schweiz
}

\author{
Permanente Honorarrückgänge und immer mehr Bürokratie prägen seit Jahren \\ den ärztlichen Alltag in Deutschland. Gesundheitsreform, Probleme bei der Er- \\ langung einer kassenärztlichen Zulassung und der sog. Gesundheitsfonds brin- \\ gen viele deutsche Arztpraxen in Existenznot. Zahlreiche deutsche Ärzte in \\ Grenznähe haben daher eine Praxis in der Schweiz eröffnet. Ihnen droht nun eine \\ abkommenswidrige Doppelbesteuerung durch deutsche Finanzämter.
}

Rainer Soboll, Bernhard Madörin

Die nachfolgende Darstellung gilt gleichermassen für Ärzte, die in einer Einzelpraxis, einer Gruppenpraxis oder einer Ärztegemeinschaft selbständig tätig sind.

Korrespondenz:

Dr. iur. Bernhard Madörin Steuerexperte, Revisionsexperte RAB meditax Fide Consult AG FMH Treuhand Services Gartenstrasse 95

CH-4002 Basel

Tel. 0612256699

bernhard.madoerin@ fmhtreuhand.ch

und

Dipl. oec. Rainer Soboll,

LL.M. CPA

Wirtschaftsprüfer, Steuerberater, Fachberater für Internationales Steuerrecht

Rainer.Soboll@ Riedlinger-Partner.de

\section{Die steuerliche Ausgangslage}

Deutsche Ärzte mit einer Praxis in der Schweiz ${ }^{1}$ sind grundsätzlich mit ihren Praxiseinkünften in der Schweiz steuerpflichtig. Gleichwohl bleiben sie, soweit sie ihren Wohnsitz in Deutschland beibehalten, weiterhin auch in Deutschland steuerpflichtig. Zur Vermeidung der zweifachen Besteuerung derselben Einkünfte hat die Schweiz mit Deutschland ein Doppelbesteuerungsabkommen (DBA) abgeschlossen. Danach steht das Besteuerungsrecht für Einkünfte aus einer in der Schweiz ausgeübten Praxis ausschliesslich der Schweiz zu; in Deutschland werden die Schweizer Praxiseinkünfte von der Besteuerung freigestellt und nur bei der Bemessung des Steuersatzes berücksichtigt.

Aufgrund des regelmässig günstigeren Schweizer Steuerniveaus konnten deutsche Ärzte durch Ausübung ihrer Tätigkeit in der Schweiz gegenüber einer freiberuflichen Berufsausübung in Deutschland als Nebeneffekt meist signifikante Steuervorteile erzielen. Diese Vorteile mussten fast zwangsläufig die Begehrlichkeiten der deutschen Finanzämter wecken.

\section{Der Besteuerungszugriff}

\section{durch das deutsche Aussensteuergesetz}

Wie viele andere Industrienationen schützt sich Deutschland vor der Verlagerung von Geschäftstätigkeiten und Steuerquellen auf Kapitalgesellschaften in niedrig besteuernde Länder durch Missbrauchsregelungen im deutschen Aussensteuergesetz (AStG). Durch die sog. Hinzurechnungsbesteuerung werden niedrig besteuerte passive Einkünfte einer von Deutschen beherrschten ausländischen Kapitalgesellschaft dem deutschen Gesellschafter unmittelbar zugerechnet und müssen von ihm - in der Regel unter Anrechnung der im Ausland bezahlten Steuer - in Deutschland versteuert werden. Dies bewirkt eine Hochschleusung der im Ausland erwirtschafteten Einkünfte auf das deutsche, höhere Steuerniveau. Diese Regelung blieb aber bis 2002 wirkungslos, wenn der deutsche
Steuerpflichtige seine Aktivitäten nicht durch eine ausländische Kapitalgesellschaft, sondern durch eine ausländische Personengesellschaft, Betriebsstätte oder Einzelpraxis betrieb.

Dem schob der deutsche Fiskus im Jahr 2003 mit der Regelung des $§ 20$ Abs. 2 AStG einen Riegel vor und regelte für alle Einkünfte, die im Rahmen einer Kapitalgesellschaft anfallen und der Hinzurechnungsbesteuerung unterliegen würden, einen Ausschluss von dem DBA-rechtlichen Freistellungsverfahren, wenn sie (u. a.) in einer ärztlichen Einzel- oder Gemeinschaftspraxis anfallen. Diese (nationale) Vorschrift verstösst ohne Zweifel gegen die Vorschriften des DBA (sog. Treaty Override). Einen solchen Verstoss gegen einen bilateralen völkerrechtlichen Vertrag hält Deutschland im Einklang mit der Auffassung des höchsten deutschen Steuergerichts, jedoch im Gegensatz zur Schweiz, für zulässig.

\section{Anwendungsvoraussetzungen \\ \$20 Abs. 2 AStC}

Die Anwendung des $\S 20$ Abs. 2 AStG auf einen in der Schweiz praktizierenden deutschen Arzt hat folgende kumulative Voraussetzungen:

- Der Arzt muss seinen Wohnsitz in Deutschland haben und seine Praxistätigkeit in der Schweiz ausüben.

- Der deutsche Arzt muss in der Schweiz nach deutschem Verständnis mit seinen Praxiseinkünften niedrig, d.h. mit weniger als $25 \%$ besteuert werden, was in der Schweiz regelmässig der Fall ist (dabei zählen Beiträge zur AHV nicht zu den Steuern).

- Der deutsche Arzt erzielt sog. schädliche passive Einkünfte, was nach dem gesetzlichen Wortlaut vorliegt, wenn der Arzt an der Erbringung der ärztlichen Dienstleistung persönlich mitwirkt.

Letzteres ist der Erbringung der ärztlichen Leistung systemimmanent. Bereits hier wird deutlich, dass die 
Regelung zu völlig verfehlten und sinnwidrigen Ergebnissen führt und ein in Deutschland ansässiger und in der Schweiz selbständig tätiger Arzt der Steuerfalle des $\S 20$ Abs. 2 AStG niemals entkommen kann.

Massnahmen der deutschen Finanzverwaltung Die bereits seit dem Jahr 2003 anwendbare Regelung des $\S 20$ Abs. 2 AStG ist in den vergangenen Jahren kaum in das Bewusstsein deutscher Steuerberater gedrungen. Auch die deutsche Finanzverwaltung hat die Anwendbarkeit dieser Vorschrift bis vor kurzem offensichtlich schlicht übersehen. Seit einigen Monaten greifen jedoch deutsche Finanzämter diese Sachverhalte - vielfach auch rückwirkend bis zum Jahr 2003 - planmässig auf. Dies erfolgt sowohl im Rahmen jährlicher Steuerveranlagungen, vielfach aber auch in Folge steuerlicher Betriebsprüfungen oder in Einspruchsverfahren. Dabei werden nicht selten durch die deutschen Finanzämter Steuernachforderungen in Höhe mehrfacher sechsstelliger Eurobeträge geltend gemacht. Eine finanzgerichtliche Überprüfung dieser Praxis steht noch aus; erste Klageverfahren sind jedoch zwischenzeitlich bereits gerichtlich anhängig²

\section{Kritik an der deutschen Position}

Obwohl die deutsche Praxis, durch nationale Rechtsvorschriften Bestimmungen des DBA auszusetzen, völkerrechtlich vertragsbrüchig ist, kann gleichwohl der betroffene deutsche Arzt die Verpflichtungen Deutschlands aus dem DBA für sich nicht klageweise einfordern, da nicht er selbst, sondern nur der andere Vertragspartner Schweiz aus dem Abkommen Rechte ableiten kann. Als Berechtigung für das Treaty Override wird durch die deutsche Finanzverwaltung vorgebracht, dass die Vertragsparteien des DBA in dessen Art. 23 ausdrücklich der jeweiligen Gegenpartei die Anwendung solcher nationalen Rechtsvorschriften zugestanden haben, die der Verhinderung der Steuerumgehung oder Steuerhinterziehung dienen.

Es bleibt hier nur die Frage, worin in dem Betrieb einer Arztpraxis grenzüberschreitend im Nachbarland auch nur der Hauch eines Missbrauchs liegen soll. Der Europäische Gerichtshof (EuGH) hat sehr treffend als Voraussetzung für den Missbrauch von Steuergesetzen das Vorliegen rein künstlicher Gestaltungen bezeichnet, die darauf ausgerichtet sind, der Anwendung bestimmter nationaler Rechtsvorschriften zu entgehen. Eine derartige Definition muss man wohl auch bei der Auslegung des DBA zugrunde legen. Bei Anlegung solcher Massstäbe ist die Eröffnung einer Praxis auf der anderen Seite des Rheins von einem Steuermissbrauch erkennbar weit entfernt.

Überhaupt erscheint die deutsche Auffassung zur Zulässigkeit eines Treaty Override zunehmend ins Wanken zu geraten. Nachdem seit vielen Jahren massgebliche Stimmen in der steuerlichen Kommentarliteratur vehement ein Treaty Override als unzulässig bekämpft haben, beginnen nun auch erste Stimmen aus der Richterschaft des Bundesfinanzhofs, sich - unter
Berufung auf entsprechende Entscheidungen des deutschen Bundesverfassungsgerichts - kritisch mit dem Treaty Override zu befassen. Dem wird man letztlich für den speziellen Fall des § 20 Abs. 2 AStG argumentativ auch nicht entgegenhalten können, dass der EuGH einen Übergang vom Freistellungs- zum Anrechnungsverfahren grundsätzlich für unbedenklich gehalten hat.

Weiterhin begegnet die Anwendbarkeit des § 20 Abs. 2 AStG insbesondere auf solche deutsche Ärzte, die ihre Praxis bereits vor der Gesetzesänderung aus dem Jahr 2003 in der Schweiz eröffnet haben, erheblichen verfassungsrechtlichen Bedenken. Der nachträgliche steuerliche Eingriff in eine langfristige vermögenswerte Disposition eines Steuerpflichtigen durch belastende steuerliche Massnahmen kann kaum mit dem Argument der sog. Abschnittsbesteuerung gerechtfertigt werden.

Die Versagung der deutschen Steuerfreistellung ärztlicher Einkünfte mit der Begründung der persönlichen Mitwirkung des Arztes an der ärztlichen Dienstleistung, erscheint auch dem Sinn und Zweck des vom Gesetzgeber Gewollten nicht zu entsprechen. Die gesetzliche Fiktion, die Praxis sei eine Kapitalgesellschaft und die Übertragung der für solche Gesellschaften geregelten Einkünftequalifikationen auf eine Arztpraxis, führen zu evident widersinnigen Ergebnissen, da die persönliche Mitwirkung des Arztes an der ärztlichen Behandlung nicht nur gewollt, sondern unabdingbar ist. Zu Recht wird in der steuerlichen Kommentarliteratur die Vorschrift des $§ 20$ Abs. 2 AStG deshalb als wenig durchdacht und in vieler Hinsicht nicht auf im Ausland erzielte ärztliche Einkünfte passend bezeichnet. Dies legt die erforderliche Beschränkung der Vorschrift auf ihren tatsächlichen Sinn und Zweck nahe.

Auch die Anknüpfung an einen Steuersatz von $25 \%$ für eine Niedrigbesteuerung ist verfehlt. Selbst in Deutschland können Kapitalgesellschaften wie auch Personen unter bestimmten Voraussetzungen Steuersätze unter $25 \%$ erreichen. Die Bundesrepublik Deutschland ist also heute bereits selbst ein «Niedrigsteuerland». Man kann wohl nur schwerlich eine in der Schweiz ausgeübte, sozial wertvolle Tätigkeit als Missbrauch abstempeln, die im eigenen Land in vielen Fällen ebenfalls «niedrig» besteuert wird. Es ist deshalb nicht gerechtfertigt, die einschneidenden Folgen des $\S 20$ Abs. 2 AStG bereits bei einem Steuersatz von $25 \%$ zu ziehen, zumal unter Ausblendung der Lasten aus der Schweizer AHV.

\section{Was können betroffene Ärzte tun?}

Betroffene Ärzte sollten den Massnahmen der deutschen Finanzämter mit allen rechtlich möglichen Mitteln entgegentreten. Entsprechende deutsche Steuerbescheide sollten durch Einspruch angefochten werden; soweit erforderlich, sollte gerichtlicher Rechtschutz in Anspruch genommen werden. gericht Baden-Württemberg unter dem Az. 3 K 427/09. 